Proceedings of the 48th International School and Conference on the Physics of Semiconductors "Jaszowiec 2019"

\title{
MnSe - Molecular Beam Epitaxy Growth and Optical Characterisation
}

\author{
J. KuchareK*, R. BożeK And W. PaCuski \\ Institute of Experimental Physics, Faculty of Physics, University of Warsaw, \\ L. Pasteura 5, PL-02093 Warszawa, Poland
}

\begin{abstract}
Here, we report on the optical properties of manganese selenide (MnSe) layers grown by molecular beam epitaxy. We compare MnSe film growths on c-plane $\mathrm{Al}_{2} \mathrm{O}_{3}$ in various conditions. Samples were analyzed in situ using reflection high energy electron diffraction. This technique confirmed that in specific conditions growth of crystals well oriented to the substrate is possible and the surface of such crystals became flat after tens of $\mathrm{nm}$, with typical grain size dependent on growth time. Further we studied samples using room temperature optical spectroscopy. Intensity of broad peak observed in photoluminescence spectra is strong for samples with large MnSe crystallites and very weak for samples with small crystallites. Origin of this peak is attributed to $\mathrm{Mn}^{2+} d-d$ transitions strongly hybridised with Se shells. Thanks to the transparency of $\mathrm{Al}_{2} \mathrm{O}_{3}$, transmittance measurements have been performed on obtained layers, revealing a broad absorption band in blue spectral range, which explains the yellow color of MnSe layers. Properties observed in transmittance and the Raman scattering - large band gap and the Raman lines close to $250 \mathrm{~cm}^{-1}$ are in agreement with the data reported for the most stable polymorph of MnSe - rocksalt structure (alpha-MnSe).
\end{abstract}

DOI: 10.12693/APhysPolA.136.598

PACS/topics: MnSe crystals, molecular beam epitaxy, Raman scattering, photoluminescence

\section{Introduction}

Monolayers of van der Waals $\mathrm{MnSe}_{2}$ provide an opportunity for exploring two-dimensional (2D) magnetism for scientific and technological advances. Thanks to recently reported room temperature ferromagnetism of a monolayer $\mathrm{MnSe}_{2}$ grown by molecular beam epitaxy (MBE) on GaSe [1], manganese selenide compounds hold promise for potential applications in energy efficient information storage and processing. On the other hand, the most stable MnSe is in rocksalt structure (alpha-MnSe) and exhibit antiferromagnetic properties [2-5]. Purpose of this work was to optimize conditions of epitaxial growth which allows for obtaining monocrystalline MnSe, which could be further a ferro- or antiferromagnetic buffer layer for growth of transition metal dichalcogenides and used for study of proximity effects. We present growth on monocrystalline $\mathrm{Al}_{2} \mathrm{O}_{3}$ substrate, which have several optical advantages, e.g., a possibility of transmittance measurements.

\section{Samples preparation}

The samples used in this study were grown in a double chamber MBE system delivered by SVT Associates. In this work we used II-VI growth chamber equipped with standard Knudsen effusion cells for both Se and Mn sources. We have grown MnSe layers with a thickness of about $100 \mathrm{~nm}$ and $200 \mathrm{~nm}$, respectively, for $45 \mathrm{~min}$ and

\footnotetext{
* corresponding author; e-mail: j.kucharek2@student.uw.edu.pl
}

90 min growth. The growth rate was determined from in situ optical reflectance oscillations. We used $c$-plane $\mathrm{Al}_{2} \mathrm{O}_{3}$ (sapphire) substrate. Just before the growth, the substrates were heated to $700{ }^{\circ} \mathrm{C}$. We have grown a series of samples in various conditions to optimize growth and optical properties. Based on observation of reflection high energy electron diffraction (RHEED) image of samples grown on $\mathrm{Al}_{2} \mathrm{O}_{3}$, the growth temperature window has been found as $350-400^{\circ} \mathrm{C}$, which were the temperatures resulting in a streaky RHEED pattern. We noted that RHEED pattern depends not only on growth temperature, but also it is significantly changing with time. Figure 1a shows $\mathrm{Al}_{2} \mathrm{O}_{3}$ substrate after initial heating and subsequent cooling to the growth temperature $\left(350^{\circ} \mathrm{C}\right)$. Figure $1 \mathrm{~b}$ shows the beginning of the growth when $\mathrm{Al}_{2} \mathrm{O}_{3}$ lines disappear and dots start to be visible suggesting 3D island growth in VolmerWeber growth mode. The next minutes are crucial for surface roughness. If the Se flux is too strong, circles are occurring, which means that growth is becoming polycrystalline, similarly to the already reported growth of $\mathrm{MnTe}$ on $\mathrm{Al}_{2} \mathrm{O}_{3}[6]$. However, for sufficiently low Se flux, as it is in the case of growth presented in Fig. 1, after tens of minutes only sharp RHEED lines are observed (Fig. 1c), suggesting growth of flat, monocrystalline surface. The longer the growth lasts, the stronger RHEED lines can be observed (Fig. 1d).

To demonstrate the effect of evolution of crystal quality with a growth time, we present here atomic force microscopy (AFM) images of two MnSe samples grown in the same conditions, with the same growth rate, but for one sample growth time (and amount of deposited material) is doubled (Fig. 2). Note that vertical scale is significantly elongated compared to horizontal scale. 

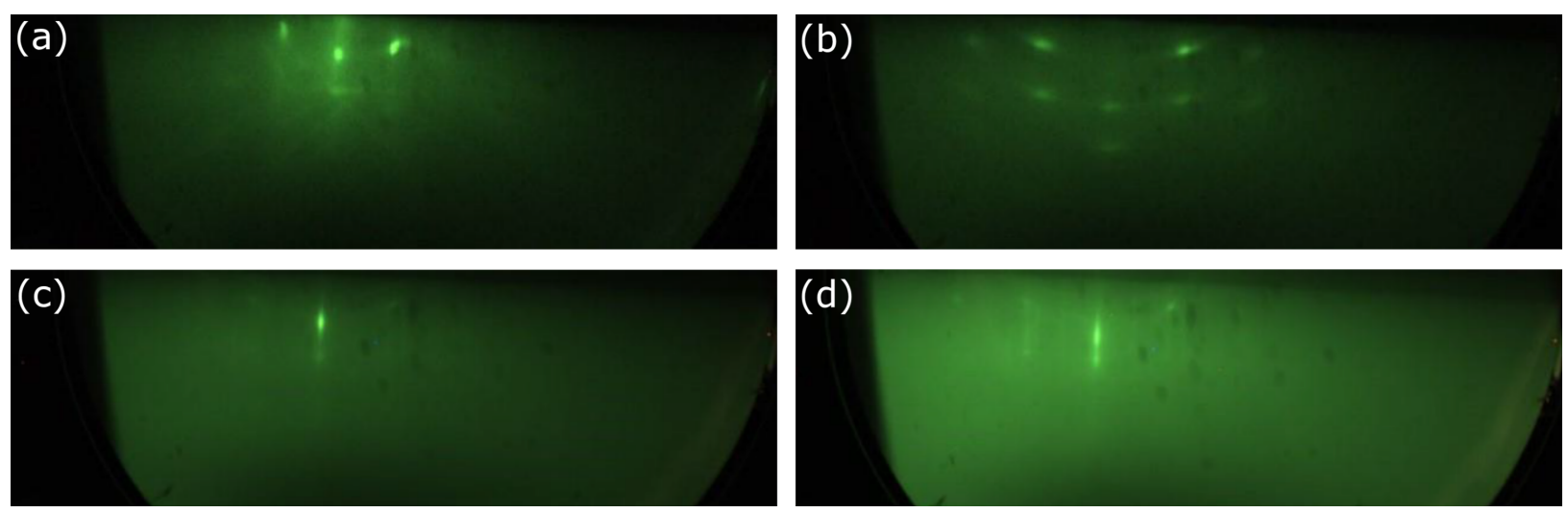

Fig. 1. RHEED patterns during the manufacturing the sample: (a) $\mathrm{Al}_{2} \mathrm{O}_{3}$ substrate before growth, (b) MnSe sample after 5 min of growth, (c) MnSe sample after 50 min of growth, (d) MnSe sample after 90 min of growth (at the end).
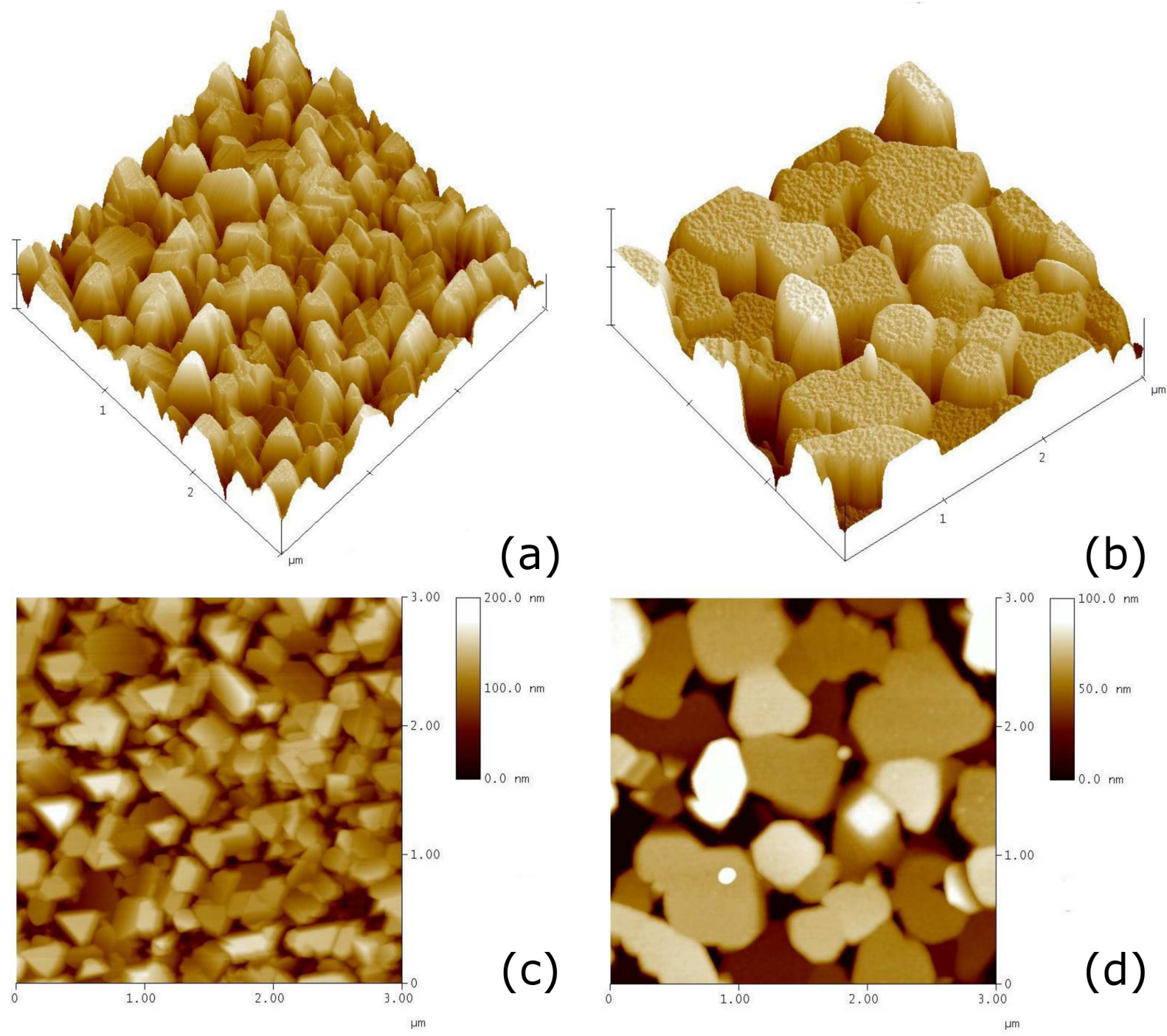

Fig. 2. AFM images of MnSe: grown on $\mathrm{Al}_{2} \mathrm{O}_{3}$ substrate. (a), (b) 3D model, (c),(d) height map. Samples shown in (a) and (c) is finished after $45 \mathrm{~min}$, samples shown in (b) and (d) is finished after 90 min. Grain boundaries exhibit relative angles closer to $60 \mathrm{deg}$ than $90 \mathrm{deg}$, so it suggests either hexagonal structure than cubic structure grown along 111 direction. 


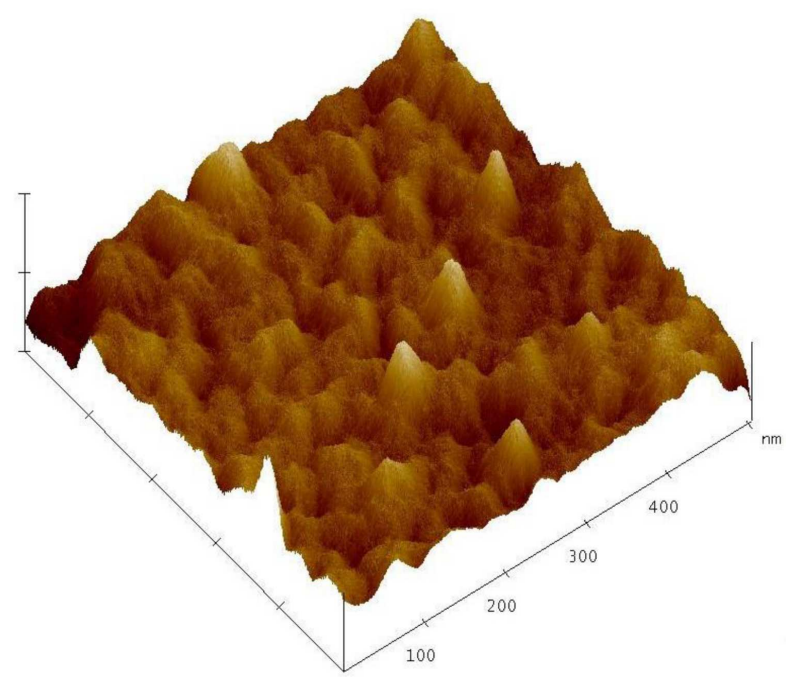

Fig. 3. AFM enlarged image of the surface of the flat part of MnSe sample shown in Fig. 2b,d. Roughness of this place of the sample is at approximately $2 \mathrm{~nm}$.
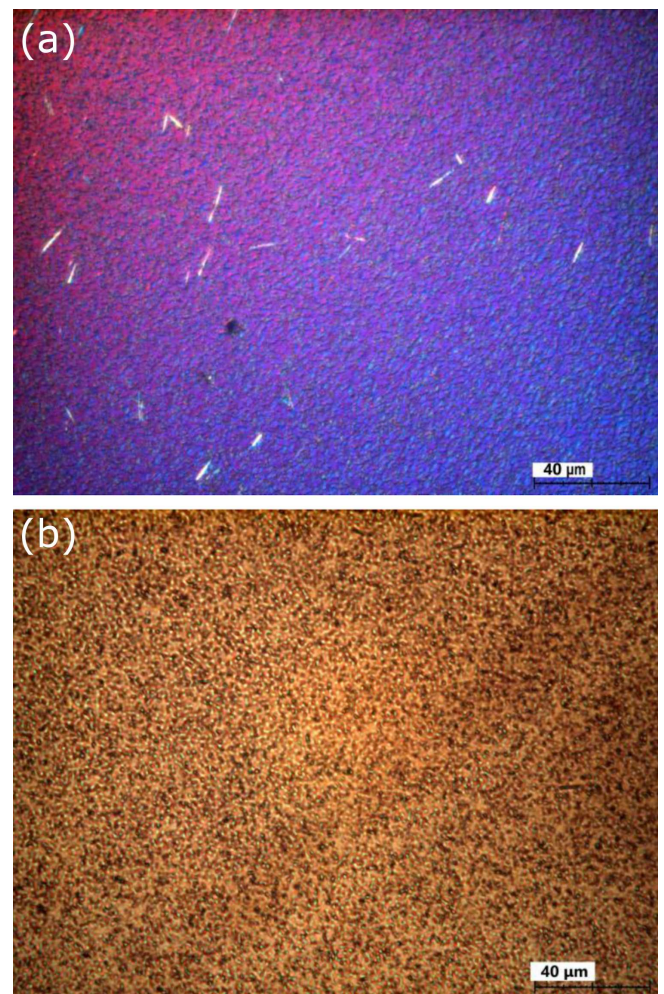

Fig. 4. Microscope images with the Nomarski contrast: (a) reflection mode, (b) transmission mode.

Samples finished after 45 min of growth exhibit high density of small size grains (order of $100 \mathrm{~nm}$ ), and only very few of them are flat on the top (Fig. 2a, c). In contrary, samples finished after 90 min contain much smaller density of grains, but several grains exceed $1 \mu \mathrm{m}$ size. Combining this with streaky RHEED pattern, we can con- clude that most of grains exhibit the same crystal orientation (defined by the substrate) and coalesce during growth leading to formation of larger grains. Even more importantly, top of almost every grain in 90 min sample is almost flat in presented scale. To zoom on surface quality of large grains, we present Fig. 3, which confirms that roughness of the large grains is approximately $2 \mathrm{~nm}$ (compared to about $100 \mathrm{~nm}$ height of grains). It is not yet an atomic flatness optimal for further growth of $2 \mathrm{D}$ materials, but it is an important hint for further development of magnetic buffers.

Since AFM images reveal about $1 \mu \mathrm{m}$ large grains, it is a natural question if they can be resolved in optical microscopes. Images obtained in transmission configuration and in reflection configuration with the Nomarski contrast are shown in Fig. 4. Grains are difficult to resolve, but clearly the light is scattered by small objects, in agreement with AFM observations.

Images from the optical microscope with the Nomarski contrast, both in reflection and transmission mode showed heterogeneity of the surface. Sample is MnSe, the same as shown in Figs. 2b,d and 3.

\section{Room temperature optical spectroscopy}

All samples were studied using room temperature Raman scattering and photoluminescence. Both were excited with $532 \mathrm{~nm}$ laser. In addition, thanks to transparent, two-side polished sapphire substrate, samples were also studied using optical transmittance. Figure 5 presents the Raman spectra of three samples grown on $\mathrm{Al}_{2} \mathrm{O}_{3}$ substrate in various conditions. For all three presented samples we observe characteristic Raman peak close to $250 \mathrm{~cm}^{-1}$. Asymmetric shape of the Raman peak suggests that it can result from two overlapping peaks. Indeed, alpha-MnSe known from literature exhibits two strong peaks in this region, at $237 \mathrm{~cm}^{-1}$ and at $256 \mathrm{~cm}^{-1}$ [4].

Figure 6 presents two photoluminescence (PL) spectra for the same series of samples as shown in Fig. 5. Sharp, double peak close to $1.79 \mathrm{eV}$ is related to $\mathrm{Cr}^{2+}$ in $\mathrm{Al}_{2} \mathrm{O}_{3}$ substrate, so it is not interesting in the present study. In contrary, broad PL peak centered close to $1.8 \mathrm{eV}$ is clearly related to MnSe. It is observed for energy slightly below well known internal $d$ - $d$ transitions of $\mathrm{Mn}^{2+}$ in diluted magnetic semiconductors, which are observed typically close to $2.1 \mathrm{eV}[7]$. One could suspect that it is emission related to the band gap, but this can be rather excluded based on transmission measurements shown in Fig. 7, where spectral region close to $1.8 \mathrm{eV}$ is almost transparent. Therefore, more reasonable explanation of PL peak is that $d$-levels of $\mathrm{Mn}^{2+}$ are mixed with shells of Se [8], and resulting optical transitions exhibit lower energy than typical $d$ - $d$ transitions of diluted $\mathrm{Mn}^{2+}$. Moreover, several Mn-related PL peaks in spectral region between $1.5 \mathrm{eV}$ and $2.4 \mathrm{eV}$ have been already studied in rock-salt MnSe [9] and zinc-blende MnTe [10] as a function of temperature to probe antiferromagnetic phase transition. 


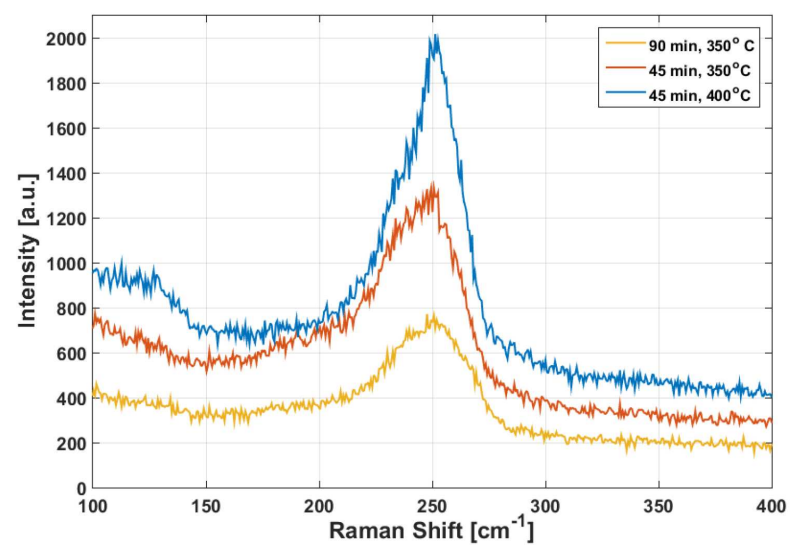

Fig. 5. Raman spectra of three MnSe samples grown on $\mathrm{Al}_{2} \mathrm{O}_{3}$ substrate in various conditions. The most intense signal (blue curve) comes from the sample grown at $400{ }^{\circ} \mathrm{C}$ (45 min of growth), its shape suggests that in fact two lines contribute to the spectrum. Red curve - sample grown at $350^{\circ} \mathrm{C}$ (45 min of growth), yellow curve - sample grown at $350^{\circ} \mathrm{C}$ (90 min of growth).

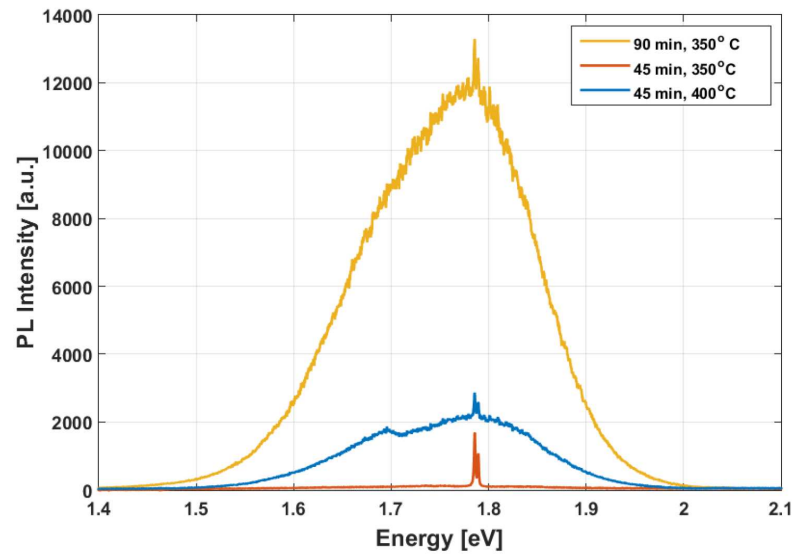

Fig. 6. Photoluminescence of three MnSe samples grown on $\mathrm{Al}_{2} \mathrm{O}_{3}$ substrate. Sharp, double peak close to $1.79 \mathrm{eV}$ is related to $\mathrm{Cr}$ in $\mathrm{Al}_{2} \mathrm{O}_{3}$ substrate. Broad luminescence is very sensitive on growth conditions - the most intense is $\mathrm{PL}$ for sample grown at $350^{\circ} \mathrm{C}(90 \mathrm{~min})$, next for sample grown $45 \mathrm{~min}$ at $400^{\circ} \mathrm{C}$. PL of sample grown only $45 \mathrm{~min}$ at $350^{\circ} \mathrm{C}$ is only hardly visible. Note that intensity of PL peaks do not correlate with intensity of the Raman peaks.

Figure 7 presents an exemplary transmittance diagram of MnSe sample grown on $\mathrm{Al}_{2} \mathrm{O}_{3}$ substrate. Transmittance measurements were performed on obtained layers, revealing broad absorption band in blue spectral range below $500 \mathrm{~nm}$, so above $2.5 \mathrm{eV}$. This explains yellow color of MnSe layers in Fig. 8 and suggests that MnSe is in a form with rather large energy gap, like the one observed for zinc blende (beta-MnSe) or typical rock salt structure (alpha-MnSe). Other forms of MnSe have much smaller bandgaps or are even metals, like $\mathrm{MnSe}_{2}$. As already noted, shape of transmittance curve does not reveal strong absorption close to energy observed in PL (about $700 \mathrm{~nm}, 1.8 \mathrm{eV}$ ). Therefore, emission in $1.8 \mathrm{eV}$ should be related to optical transition with smaller oscillator strength, e.g. $d-d$ transitions of Mn hybridised with Se shells. Such transitions have very weak oscillator strengths, resulting in weak absorptions that are difficult to observe on very thin samples.

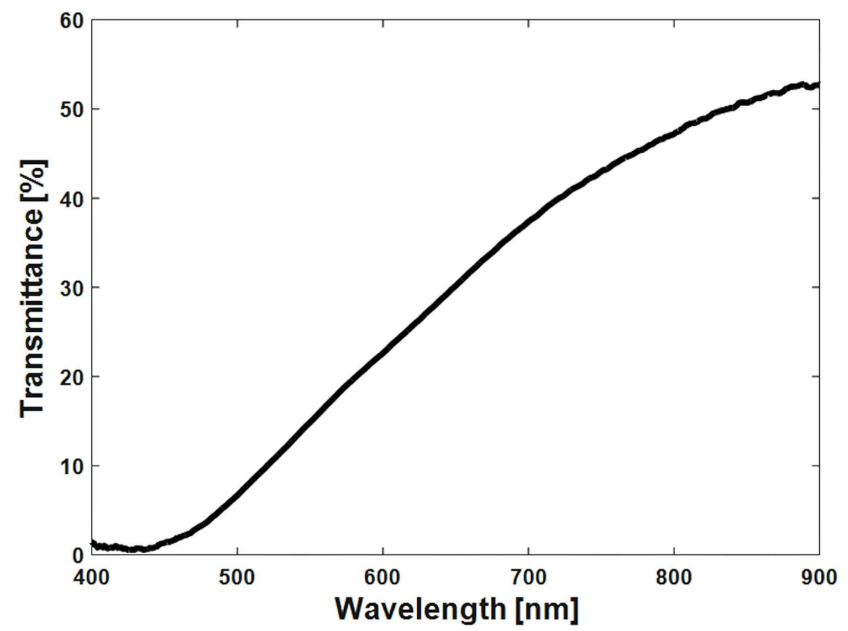

Fig. 7. Exemplary transmittance of MnSe sample grown on $\mathrm{Al}_{2} \mathrm{O}_{3}$ substrate.

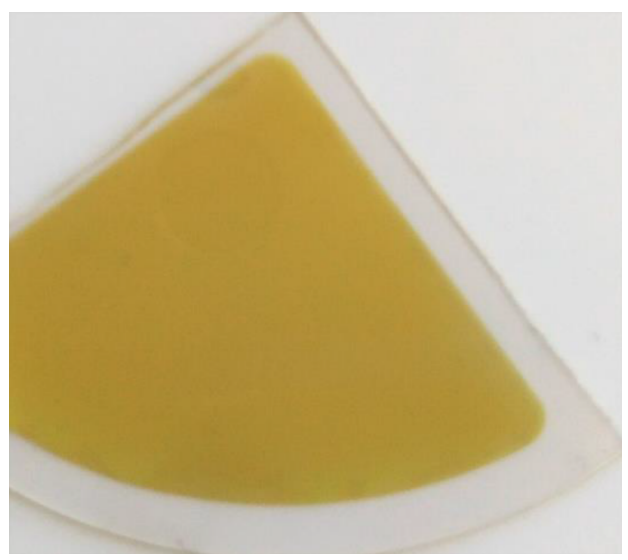

Fig. 8. Photo of MnSe sample showing yellow color, in agreement with transmittance curve.

\section{Conclusions}

We presented the possibility of epitaxial growth of MnSe on sapphire. AFM images reveal that growth leads to formation of $\mu \mathrm{m}$ size grains which are flat on top if growth is performed long enough. Use of sapphire allows for efficient optical study: the Raman scattering, PL and transmittance. Obtained results suggest that we observe growth of MnSe in most typical and stable and rock salt structure, and since it is epitaxy on $c$-plane sapphire, and 
AFM images reveals mostly 60 deg between grain boundaries, we conclude that mostly we observe MnSe grown along 111 direction, but the presence of other phases cannot be excluded based on actual results.

\section{Acknowledgments}

This work was supported by the Polish National Science Centre under decisions DEC2015/18/E/ST3/00559, DEC-2017/27/B/ST5/02284, and DEC-2016/23/B/ST3/03437. The project was carried out with the use of CePT, CeZaMat, and NLTK infrastructures financed by the European Union the European Regional Development Fund within the Operational Programme "Innovative economy".

\section{References}

[1] D.J. O'Hara, T. Zhu, A.H. Trout, A.S. Ahmed, Y.K. Luo, C.H. Lee, M.R. Brenner, S. Rajan, J.A. Gupta, D.W. McComb, R.K. Kawakami, Nano Lett. 18, 3125 (2018).

[2] R. Lindsay, Phys. Rev. 84, 569 (1951).
[3] E.D. Jones, Phys. Rev. 151, 315 (1966).

[4] A. Milutinović, N. Tomić, S. Dević, P. Milutinović, Z.V. Popović, Phys. Rev. B 66, 012302 (2002).

[5] Z.V. Popović, A. Milutinović, Phys. Rev. B $\mathbf{7 3}$, 155203 (2006).

[6] E. Przeździecka, E. Dynowska, W. Paszkowicz, W. Dobrowolski, H. Kepa, C.F. Majkrzak, T.M. Giebultowicz, E. Janik, J. Kossut, Thin Solid Films 516, 4813 (2008).

[7] W. Pacuski in: Introduction to the Physics of Diluted Magnetic Semiconductors, Springer Series in Materials Science, Eds. J.A. Gaj, J. Kossut, Vol. 144, Springer, 2010, p. 37.

[8] H. Sato, S. Senba, H. Okuda, M. Nakateke, A. Furuta, Y. Ueda, M. Taniguchi, A. Tanaka, T. Jo, J. Electron. Spectrosc. Relat. Phenom. 88, 425 (1998).

[9] A. Milutinović, Z.V. Popović, N. Tomić, S.D. Dević, Mater. Sci. Forum 453, 299 (2004).

[10] Xuanting Zhu, Qin Lian, Pengzhan Zhang, Wei Bai, Kai Tang, Liangqing Zhu, Jing Yang, Yuanyuan Zhang, Xiaodong Tang, Junhao Chu, Opt. Lett. 43 , 5547 (2018). 